\title{
CMEARTICLE
}

\section{Clinics in diagnostic imaging (201)}

Timothy Shao Ern $\underline{T a n}^{1}$, MBChB, Sook Chuei Wendy Cheong ${ }^{1}$, MBBS, FRCR, Tien Jin $\underline{T a n}^{1}$, MBBS, FRCR
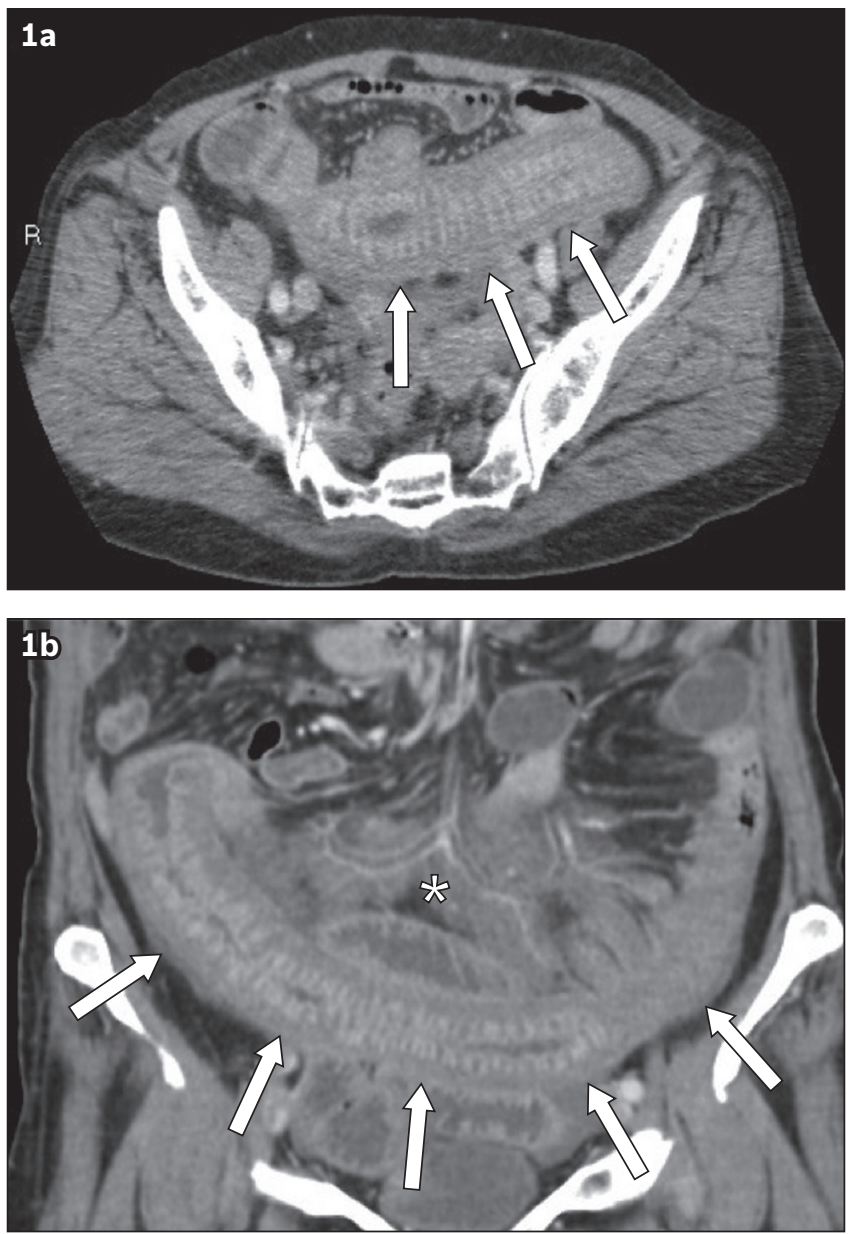

Fig. 1 (a) Axial and (b) coronal CT images of the abdomen and pelvis.

\section{CASE PRESENTATION}

A 74-year-old woman who was receiving anticoagulation therapy with warfarin for a past medical history of chronic atrial fibrillation presented to the emergency department with severe acute abdominal pain associated with diarrhoea and vomiting. The patient denied any haematemesis or melaena. Clinical examination revealed mild tenderness over the periumbilical area without guarding. The patient was afebrile and her vital signs were as follows: heart rate of 97 beats per minute, blood pressure of $97 / 69 \mathrm{mmHg}$ and oxygen saturation of $98 \%$ on room air. Initial laboratory workup revealed an international normalised ratio (INR) of $>10$ (therapeutic range for oral anticoagulants: 2-3.5) and prolonged prothrombin time of $>100.0$ (normal range 9.2-11.2) seconds. There was no acute drop in haemoglobin levels. In view of the persistent abdominal pain, contrast-enhanced computed tomography (CT) of the abdomen and pelvis (Fig. 1) was performed. What do the CT images show? What is the diagnosis? 


\section{IMAGE INTERPRETATION}

Axial (Fig. 1a) and coronal (Fig. 1b) CT images of the abdomen and pelvis demonstrate concentric hyperdense mural thickening in a distal jejunal loop (arrows) with adjacent high-density stranding in the small bowel mesentery (asterisk), in keeping with acute small bowel intramural haematoma. Features of developing reactive ileus of the small bowel loops proximal to the affected jejunal segment were also noted on imaging (not shown in Fig. 1). No evidence of pneumatosis intestinalis or intraperitoneal free gas was seen.

\section{DIAGNOSIS}

Small bowel intramural haematoma induced by anticoagulation therapy with associated reactive ileus.

\section{CLINICAL COURSE}

The patient was managed conservatively with simple analgesia, cessation of her warfarin treatment and reversal therapy with $0.5 \mathrm{mg}$ of intravenous vitamin K. No transfusion of blood products was required. The patient's symptoms resolved after a few days and warfarin $2 \mathrm{mg}$ was recommenced upon discharge following three days of hospitalisation with optimal INR control.

\section{DISCUSSION}

Non-traumatic spontaneous intramural small bowel haematoma, once considered a rare complication of oral anticoagulation (OAC) therapy, is being reported with increasing frequency. In adults, anticoagulation with warfarin is the most common cause of spontaneous intramural small bowel haematoma with an incidence of one in 2,500 patients on warfarin ${ }^{(1)}$ and predominantly affects males, with an average age of presentation of 57.6 years. ${ }^{(2)}$ This condition has also been described as a complication of bleeding disorders, malignancies, vasculitis and, less commonly, endoscopic biopsies and injection therapies. Spontaneous intramural small bowel haematoma due to anticoagulation with heparin has also been described in one paediatric patient who received therapeutic doses of lowmolecular-weight heparin for deep venous thrombosis. ${ }^{(3)}$

As spontaneous intramural small bowel haematoma is still relatively uncommon, there are few case reports describing this clinical entity. The largest series included 13 patients (mean age of presentation 64 years), of whom eight received OAC.(4) All patients presented with abdominal pain, and small bowel obstruction was present in 11 (85\%) of the patients. The jejunum was the most common site of the haematoma, followed by the ileum and duodenum. The mean INR at presentation was 11.6.

Other risk factors for developing spontaneous small bowel haematoma include haemophilia, idiopathic thrombocytopenic purpura, leukaemia, lymphoma, myeloma, chemotherapy, vasculitis, pancreatitis and pancreatic cancer. ${ }^{(5)}$ In a study investigating the occurrence and predictive factors of excessive warfarin anticoagulation (defined as INR $\geq 9$ ) in 13,618 patients with atrial fibrillation, excessive alcohol consumption and impaired renal function were found to be significant permanent risk factors, while recent antibiotic or antifungal medication use, the first six months of warfarin treatment, and recent hospitalisation were significant temporary risk factors. ${ }^{(6)}$

In up to $85 \%$ of cases of spontaneous intramural intestinal haematoma in patients on anticoagulation, the small bowel is most commonly involved, in particular the jejunum, where bleeding from small vessels commonly occurs in the submucosa. ${ }^{(7)}$ However, it remains unclear why the jejunum is the site of predilection for spontaneous haematoma. In affected patients, it has been postulated that the progression of symptoms is due to an intramural osmotic gradient caused by the haematoma, resulting in intestinal wall expansion. ${ }^{(8)}$ Extension of small bowel haematomas into the colon is rare, as it has been hypothesised that the taeniae coli may protect against the initiation or expansion of the haemorrhage. Duodenal haematomas are more common in children and cases of blunt trauma, in which the relatively fixed retroperitoneal position of the duodenum along with its rich submucosal vascular supply and close anterior relationship to the lumbar spine are predisposing factors. ${ }^{(5)}$ The oesophagus is a rare location for intramural haematoma, with nearly all cases presenting with chest pain and mostly associated with achalasia. ${ }^{(9)}$ Other causes include oesophageal instrumentation, injection therapy and ingestion of foreign bodies. Rectal intramural haematomas have been reported in cases of traumatic insertion of foreign bodies, injection therapy or stapled haemorrhoidectomy. ${ }^{(10)}$

The clinical presentation of spontaneous intramural small bowel haematoma varies widely and can range from mild vague abdominal pain to intestinal obstruction and even an acute abdomen. Nausea and vomiting are related to high intestinal obstruction involving the duodenum and/or proximal jejunum. Often, the diagnosis is established only after abdominal imaging or an exploratory laparotomy is performed. Gastrointestinal haemorrhage may result from a ruptured intramural haematoma, although this is rare. In some cases, peritoneal irritation may be present, indicating the development of severe complications such as bowel necrosis, perforation or haemoperitoneum.

Theoretically, spontaneous bleeding in patients on anticoagulation can possibly occur in any part of the body, especially in the elderly. Axial or appendicular spontaneous intramuscular haematomas may occur, commonly involving the rectus sheath of the anterior abdominal wall (Fig. 2), chest wall (Fig. 3) and iliopsoas (Fig. 4), where bleeding may arise from the epigastric vessels; internal thoracic, lateral thoracic or thoracodorsal vessels; and lumbar vessels, respectively. ${ }^{(11,12)}$ Other less common sites of involvement include the gluteal regions and extremities, where haematomas may lie within the muscular compartments or subcutaneous soft tissue (Fig. 5). Haematomas occurring within muscular compartments of the extremities are contained by the overlying fascia and if they are large, can result clinically in compartment syndrome. Soft tissue haematomas of the abdominal wall may diffuse into the peritoneal or retroperitoneal space.

Spontaneous haemorrhage may also arise anywhere along the urinary tract in patients on anticoagulants, who often present with abdominal pain and haematuria (Fig. 6). ${ }^{(13)}$ In patients with 

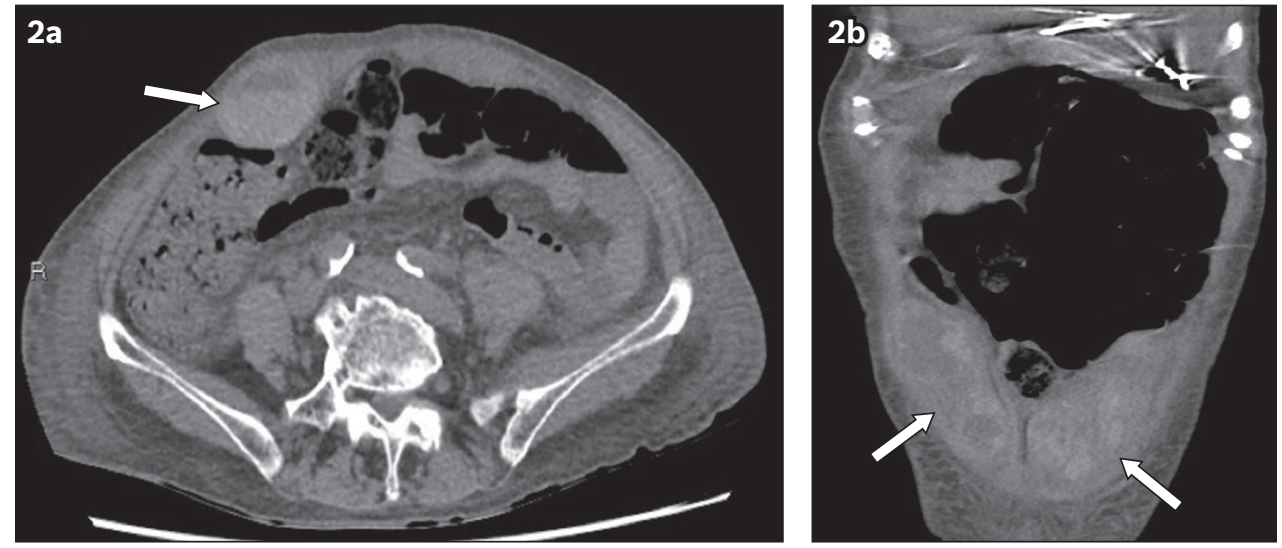

Fig. 2 A 75-year-old woman on warfarin therapy for atrial fibrillation presented with abdominal pain. (a) Axial and (b) coronal CT images of the abdomen show a large abdominal wall haematoma (arrows). Laboratory workup revealed an acutely low haemoglobin level and prolonged prothrombin time of 39.2 seconds and international normalised ratio of 3.60 .
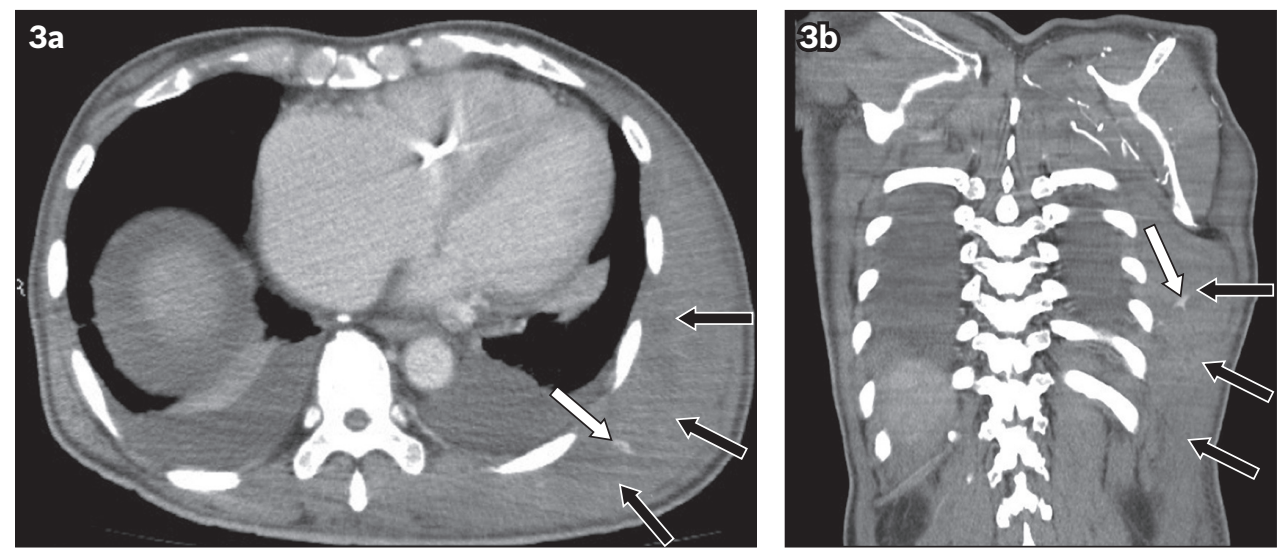

Fig. 3 A 61-year-old man with mitral valve stenosis and prior cerebrovascular disease who was on warfarin and ticlopidine therapy presented with soft tissue swelling and ecchymosis of the left chest wall. Laboratory workup revealed a prolonged international normalised ratio of 3.17. (a) Axial and (b) coronal thoracic CT images show a left posterolateral chest wall haematoma (black arrows). There is a small blush of contrast within the chest wall haematoma (white arrows) indicating a focus of active haemorrhage.
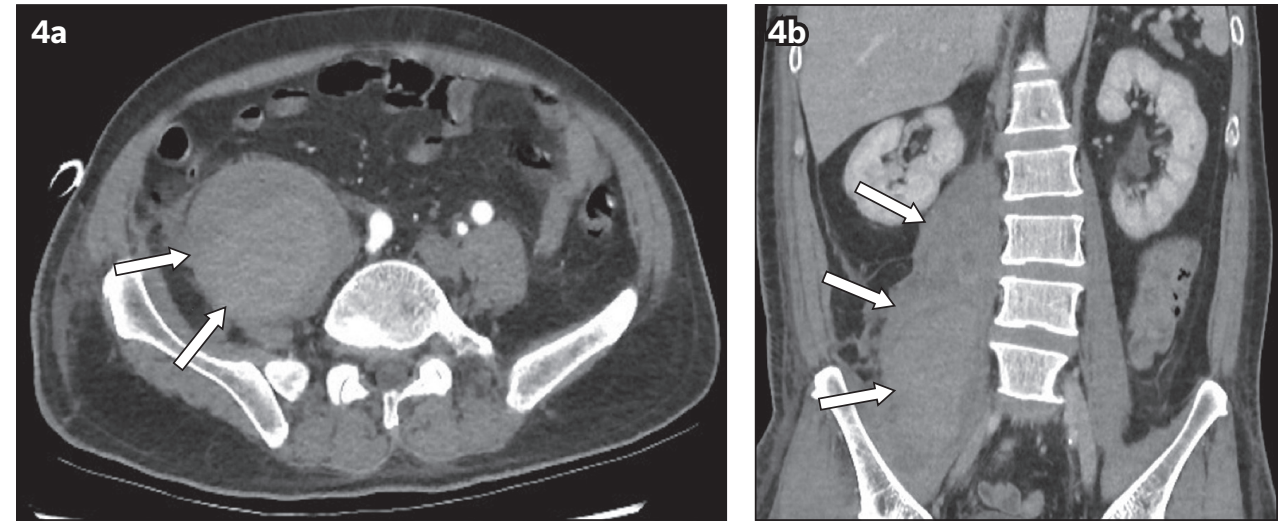

Fig. 4 An 82-year-old woman on warfarin therapy for mitral stenosis and atrial fibrillation with associated drop in haemoglobin level and prolonged coagulation profile (international normalised ratio 7.32, prothrombin time 74.2 seconds, activated partial thromboplastin time 61.0 seconds) presented with right hip and flank pain. (a) Axial and (b) coronal CT images of the abdomen show an acute right psoas haematoma (arrows).

pre-existing renal cysts, spontaneous haemorrhage into these cysts may also be induced by anticoagulation, which can then lead to the development of perinephric or retroperitoneal haematomas (Fig. 7). Suburothelial haemorrhage within the ureters or urinary bladder may also occur in patients on anticoagulation. In the presence of relevant risk factors, other sinister causes of haematuria, such as an underlying urothelial malignancy, should be excluded with thorough urological evaluation.
Spontaneous retroperitoneal haemorrhage, also known as Wunderlich syndrome, is another serious complication of anticoagulation that can develop in the presence of bleeding renal tumours or cysts, severe portal hypertension, vascular aneurysms and inflammatory vascular disorders (Fig. 8). ${ }^{(14)}$ Unlike haemorrhage in other locations, retroperitoneal bleeding can be difficult to diagnose clinically due to an absence of localising signs and symptoms. This may result in a delay in its diagnosis and 

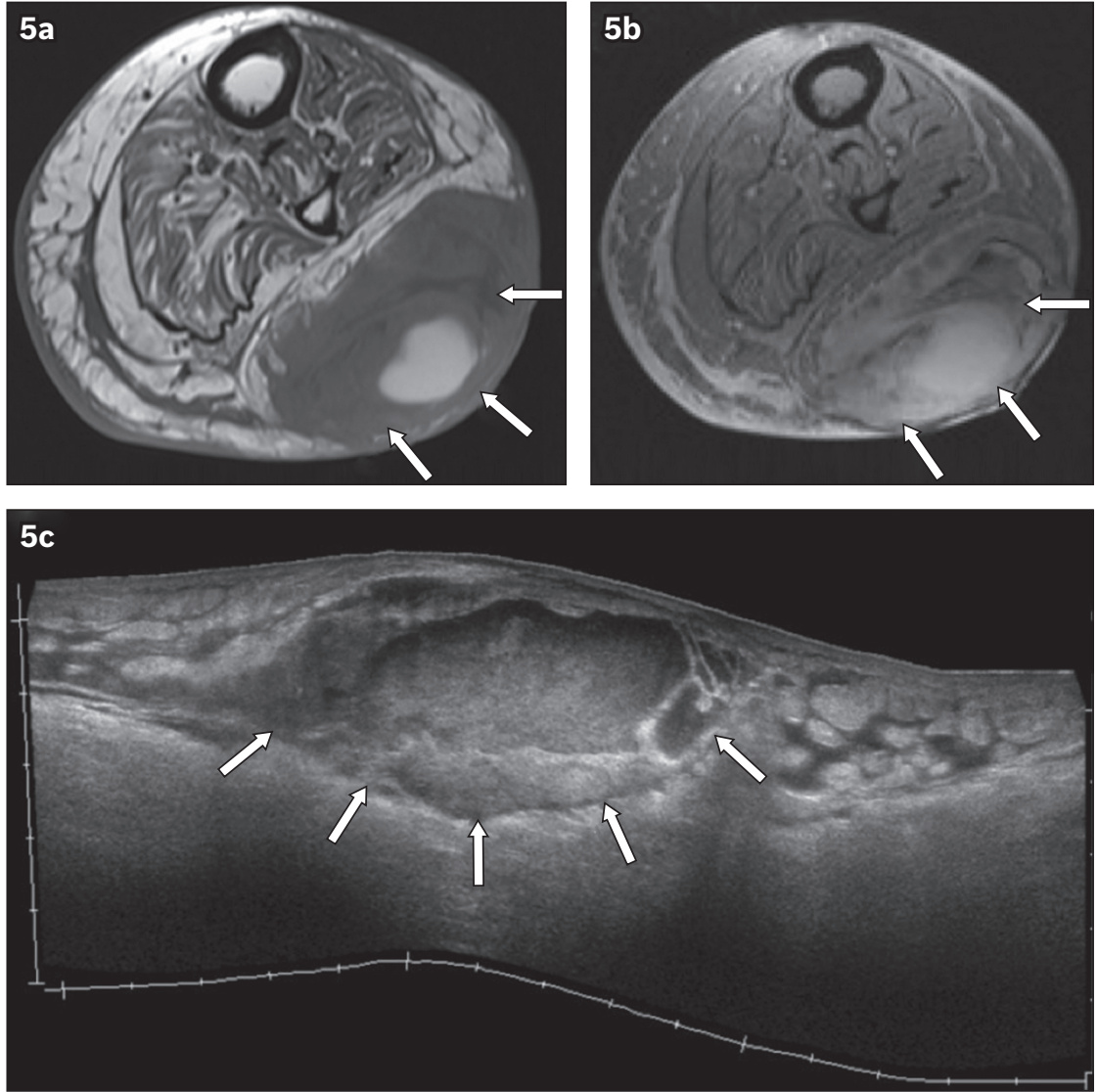

Fig. 5 An 87-year-old woman presented with acute left calf swelling and pain following warfarin therapy for left lower limb deep vein thrombosis. Coagulation parameters were prolonged, with an international normalised ratio of 3.25 and prothrombin time of 31.6 seconds. Axial (a) T1-W and (b) gradient echo MR images as well as (c) US image of the left calf show a large heterogeneous, subcutaneous left calf haematoma (arrows).
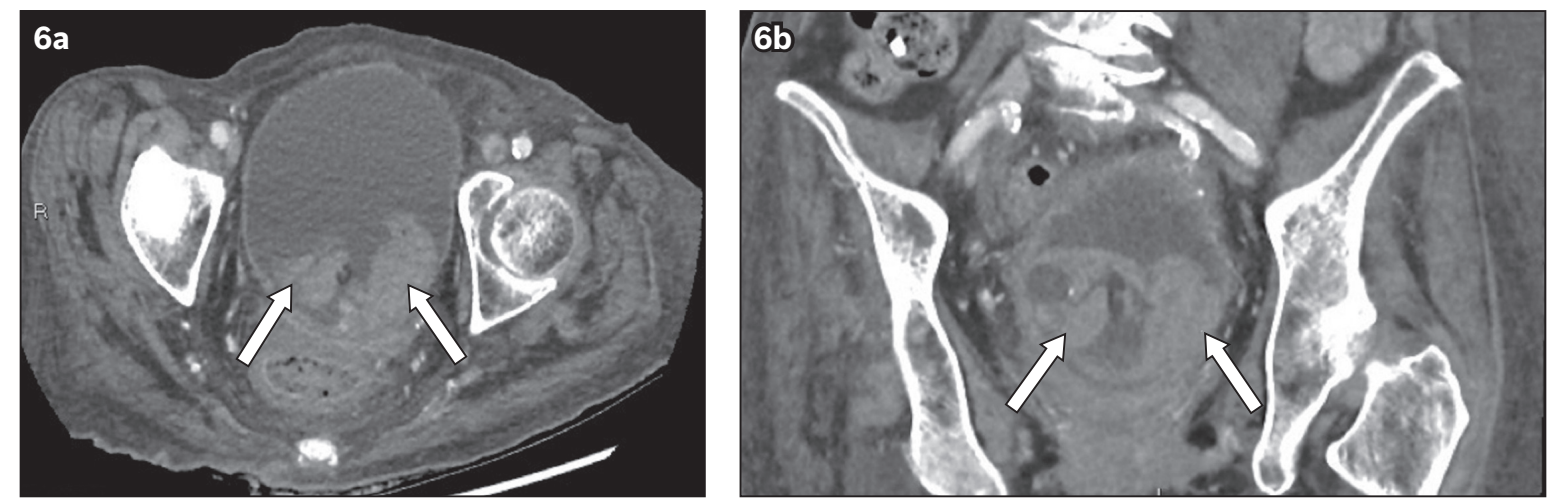

Fig. 6 A 68-year-old man was on warfarin therapy for atrial fibrillation, with raised international normalised ratio of 3.50 and prolonged prothrombin time measuring 38.4 seconds at the time of clinical presentation. (a) Axial and (b) coronal CT images of the pelvis show gross haematuria within the urinary bladder (arrows).

management, hence leading to significant morbidity or death. ${ }^{(15)}$ The retroperitoneum is considered a critical site for bleeding due to possible compression of vital neurovascular structures, resulting in compromise of the function of surrounding organs and lumbosacral plexopathy.

Spontaneous intracranial haemorrhages ( $\mathrm{ICHs})$, comprising the intraparenchymal, subdural, extradural and subarachnoid subtypes (Fig. 9), are also considered to be critical. They are the most feared complication of anticoagulation due to their serious clinical consequences of coma, permanent neurological deficits and even death, with a high 30-day mortality rate of close to $50 \%{ }^{(16)}$ As approximately $20 \%$ of spontaneous $\mathrm{ICH}$ is attributed to anticoagulation, a careful approach to restarting anticoagulants after an ICH should be tailored for each patient, weighing the risks and benefits for each individual. Ongoing anticoagulation also features as one of the general predictive factors of $\mathrm{ICH}$ recurrence along with the mechanism of $\mathrm{ICH}$ (i.e. spontaneous vs. traumatic), location of the initial bleed (which may point to the presence of underlying hypertension), the presence of amyloid angiopathy, and the overall number of microbleeds. ${ }^{(17)}$

$\mathrm{CT}$ is the imaging modality of choice for bleeding manifestations induced by anticoagulation. In intramural intestinal haematomas, characteristic features include circumferential bowel wall thickening, intramural hyperdensity and luminal narrowing 

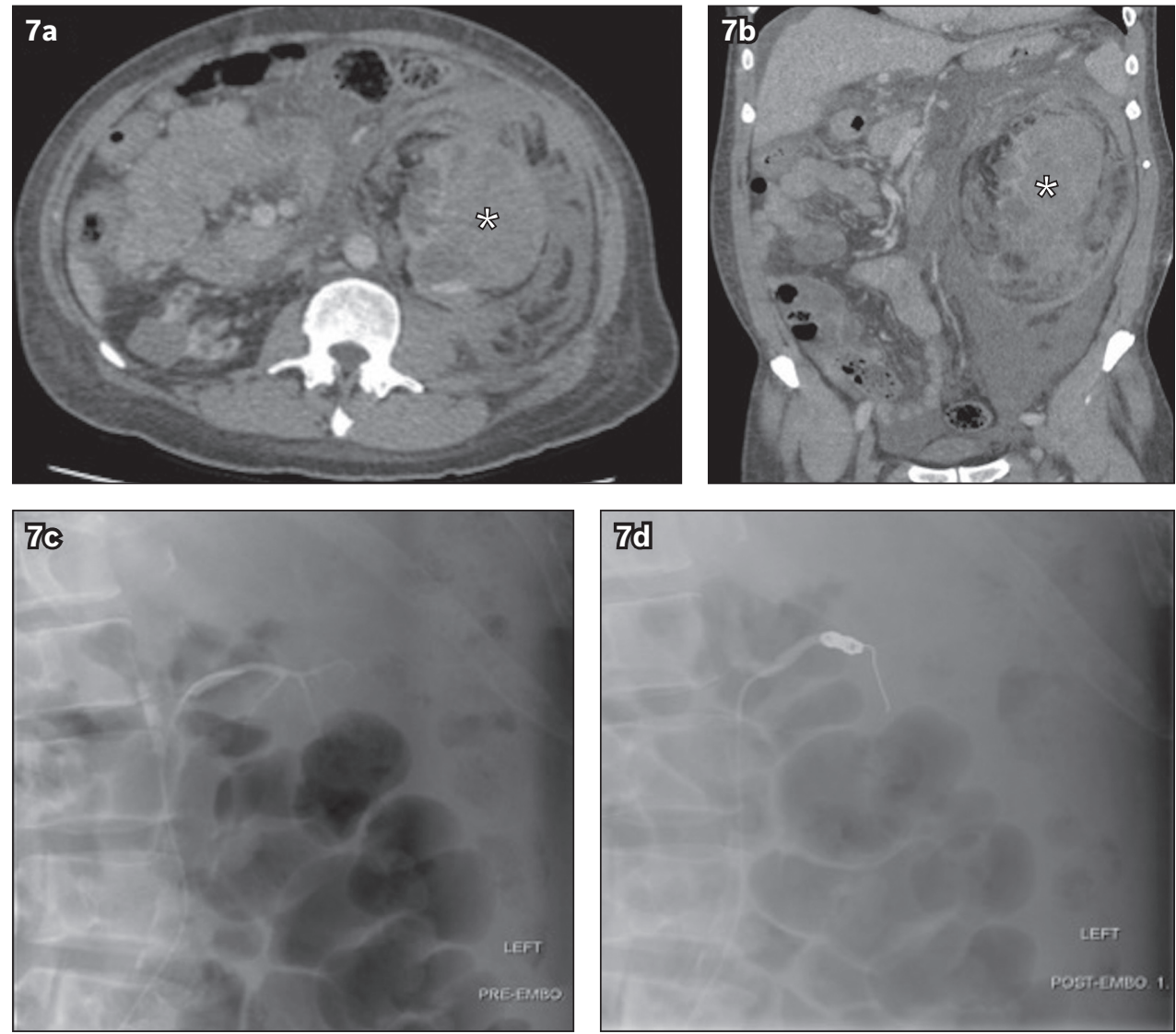

Fig. 7 A 66-year-old man on warfarin therapy for atrial fibrillation presented with acute left flank pain, low haemoglobin level of $6.6 \mathrm{~g} / \mathrm{dL}$, slightly prolonged prothrombin time of 11.6 seconds and subtherapeutic international normalised ratio of 1.11. (a) Axial and (b) coronal abdominopelvic CT images show a large left perinephric haematoma (asterisks) secondary to a ruptured left haemorrhagic renal cyst with retroperitoneal haematoma. The patient subsequently underwent digital subtraction angiography of the single left renal artery. Angiograms show (c) no obvious contrast blush; however, (d) two $6 \mathrm{~mm} \times 14 \mathrm{~mm}$ Nester coils were deployed into the left renal artery prophylactically.
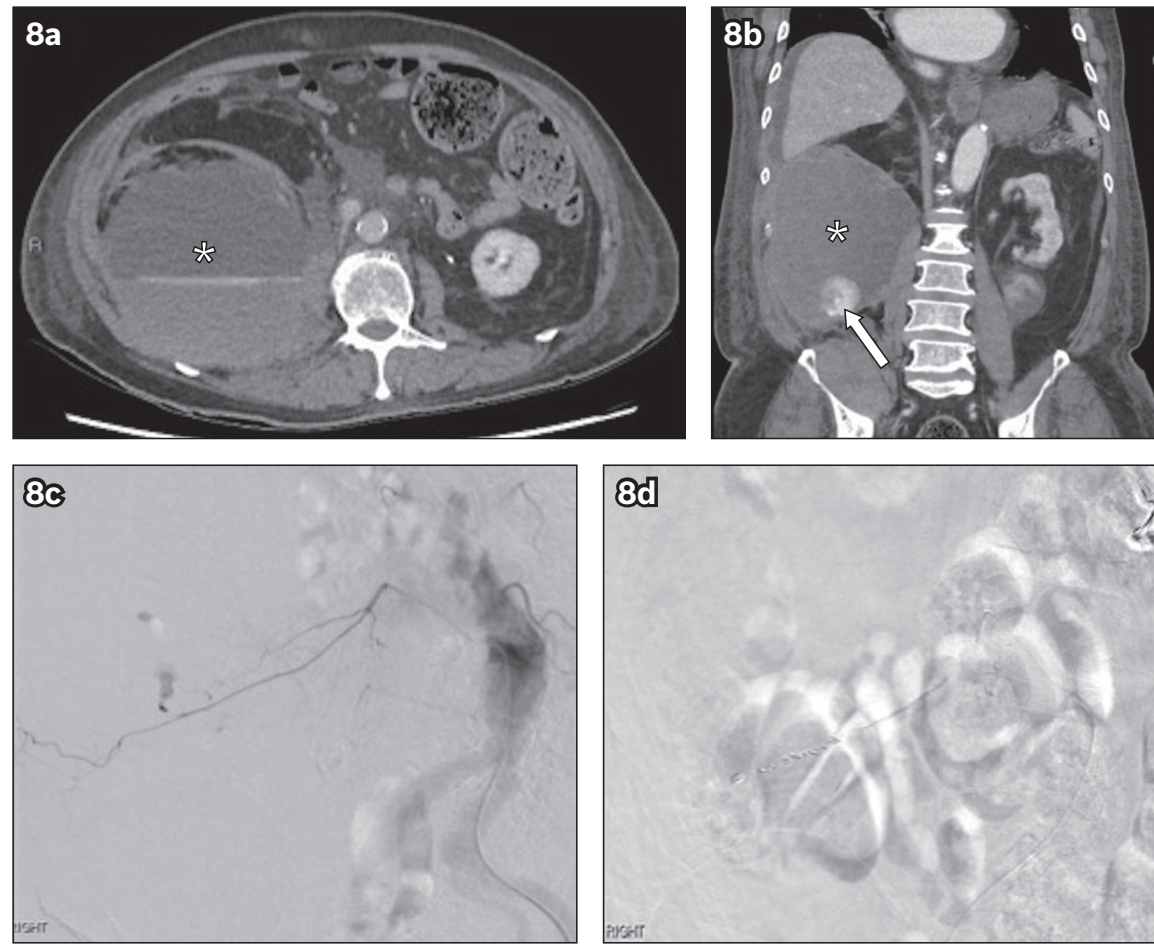

Fig. 8 An 80-year-old man on warfarin therapy for atrial fibrillation presented with low back pain and hypotension. His activated partial thromboplastin time and prothrombin time were prolonged at 35.7 seconds and 12.5 seconds, respectively. (a) Axial and (b) coronal abdominopelvic CT images show large right retroperitoneal haematoma (asterisks). There is a blush of contrast within the inferior aspect of the haematoma (arrow), indicating a focus of active haemorrhage. The patient subsequently underwent digital subtraction angiography. Angiograms show (c) blush at the right $L 3$ lumbar vertebral artery in a contrast run, which was in keeping with active haemorrhage; and (d) successful occlusion of the bleeding vessel with one $2 \mathrm{~mm} \times 3 \mathrm{~cm}$ and two $3 \mathrm{~mm} \times 5 \mathrm{~cm}$ MicroNester coils. 

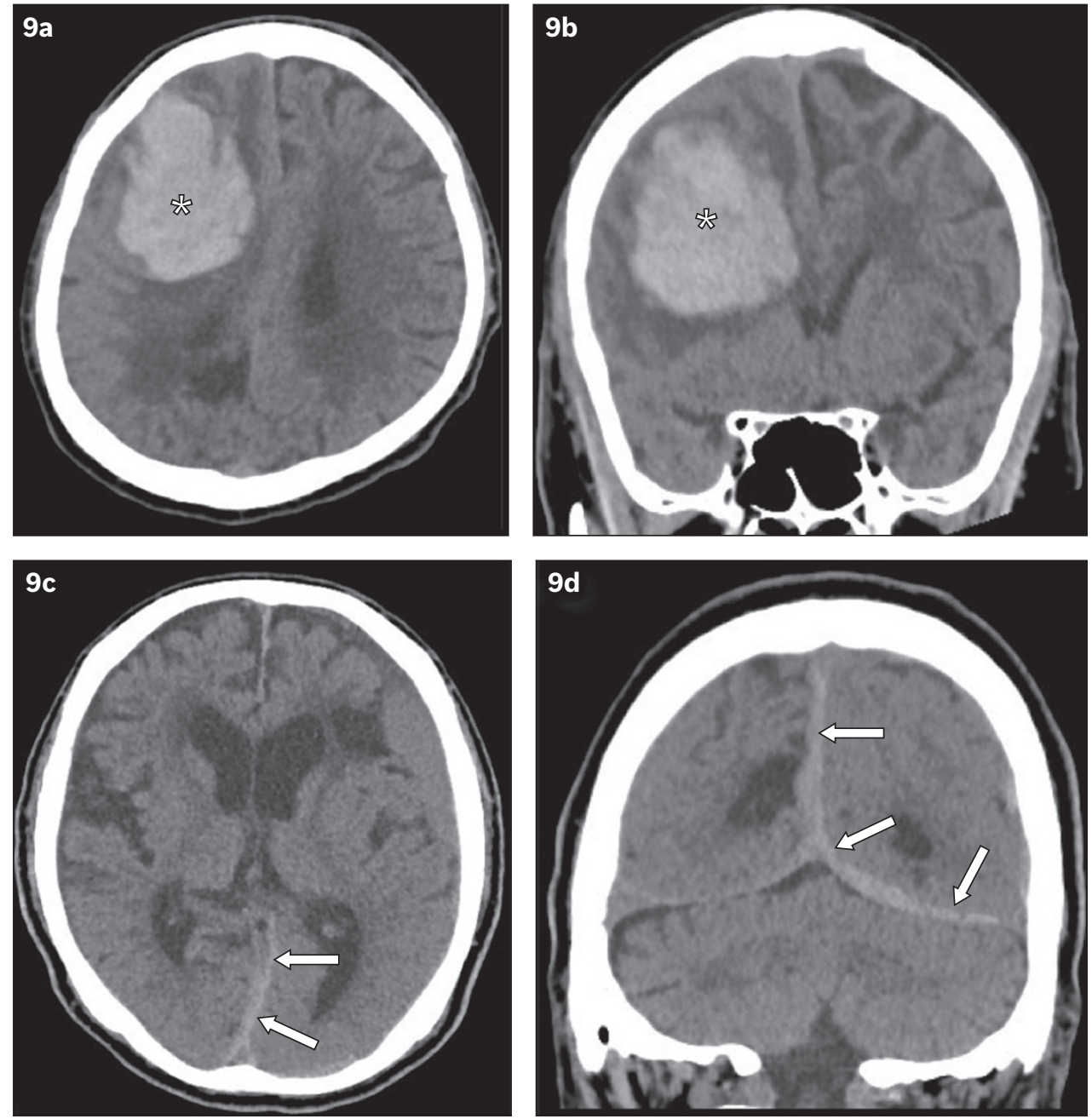

Fig. 9 (a \& b) A 69-year-old man on lifelong warfarin therapy (international normalised ratio [INR] 2.34, activated partial thromboplastin time 40.7 seconds, prothrombin time 24.9 second) for aortic and mitral valve replacements presented with left-sided weakness. Non-contrast enhanced (a) axial and (b) coronal CT images of the brain show a large acute right frontoparietal parenchymal haematoma (asterisks). (c \& d) A 64-year-old man on lifelong warfarin therapy (INR 5.26) for atrial fibrillation presented with a severe headache. Non-contrast enhanced (c) axial and (d) coronal CT images of the brain show acute subdural haematoma (arrows) along the cerebral falx and left cerebellar tentorium.

that may be accompanied by intestinal obstruction. ${ }^{(4,18)}$ It has been proposed that non-contrast CT may be performed before the acquisition of contrast-enhanced images to demonstrate the presence of any intramural haemorrhage that may be masked by contrast enhancement. (1) $\mathrm{CT}$ angiogram is useful in detecting active haemorrhage by identifying the presence of contrast extravasation and can also help to guide embolisation therapy. In our index case, contrast-enhanced CT alone was sufficient to make the diagnosis. On ultrasonography, an acute intramural small bowel haematoma may present with intestinal wall thickening involving mainly the submucosal layer, although this finding on its own is strictly non-specific. In spontaneous bowel haematomas, the involved bowel segments are usually longer than in traumatic haematomas with an average length of $23 \mathrm{~cm} .{ }^{(4)}$ Compared to other conditions such as malignancy, inflammatory or ischaemic bowel disease, bowel imaging findings often normalise within two months after the onset of symptoms.

On magnetic resonance (MR) imaging, the concentric ring sign is pathognomonic for a subacute duodenal haematoma. Varying signal intensities are seen at the peripheral outer, inner and central components of the haematoma, attributable to the paramagnetic properties of iron in the haemoglobin degradation products within the haematoma. ${ }^{(7)}$ However, the MR imaging characteristics of intramural haematomas in other parts of the small bowel are not as well defined. The role of endoscopy in diagnosing intramural intestinal haematomas is not well established, as most cases are diagnosed on imaging. However, endoscopy may aid in relieving obstructive symptoms via needleknife incision of the duodenal haematoma. ${ }^{(19)}$

According to the expert consensus of the American College of Cardiology (ACC), initial management of bleeding in patients with OAC usually involves discontinuation of the offending anticoagulant and reversal of its effects with the relevant reversal agents. ${ }^{(20)}$ In almost all cases of non-life-threatening spontaneous intramural intestinal haematoma, improvement of symptoms usually occurs within 4-6 days and the haematoma is expected to resolve within two months from the onset. ${ }^{(19)}$ Moreover, re-initiating anticoagulant therapy appears to be safe once bleeding has been controlled (including patients who had no discrete bleeding source identified) and/or after resolution of the haematoma, as long as the therapeutic range for coagulation parameters is met. In a systematic review of observational studies, 
a lower risk of thromboembolism and death was reported in patients with OAC-associated gastrointestinal bleeding who resumed anticoagulation compared to those who did not, but with a non-significant increase in the risk of recurrent bleeding. ${ }^{(21)}$ The timing of anticoagulation re-initiation has not been systematically studied and is highly variable. However, in patients with atrial fibrillation, restarting warfarin $>7$ days after a bleed is associated with improved survival and reduced thromboembolic events without an increased risk of gastrointestinal bleeding recurrence. ${ }^{(22)}$ In the context of $\mathrm{ICH}$, limited data exists on re-initiation of OAC. Depending on bleed characteristics, risk factor modification and clinical indications for anticoagulation, restarting OAC after a non-lobar ICH may be considered. There is a particularly high risk of re-bleeding if underlying amyloid angiopathy is present. As such, resuming anticoagulation in these situations should involve consultation with either neurology or neurosurgical expertise. The ACC guidelines favour resumption of anticoagulation at least four weeks from an $\mathrm{ICH}$ event in patients without high thrombotic risk.

As with trauma patients, transfusion of blood products is usually indicated in major bleeds that result in haemodynamic compromise or a haemoglobin drop $\geq 2 \mathrm{~g} / \mathrm{dL}$. Patients with symptomatic anaemia or active bleeding should receive red blood cell transfusions to maintain haemoglobin $\geq 7 \mathrm{~g} / \mathrm{dL}$. ${ }^{(23)}$ A target haemoglobin level $\geq 8 \mathrm{mg} / \mathrm{dL}$ has been recommended in patients with underlying coronary artery disease. Platelets and cryoprecipitate transfusions may be instituted with the aim of maintaining a platelet count $\geq 50 \times 10 \% / \mathrm{L}$ and a fibrinogen level $>100 \mathrm{mg} / \mathrm{dL}$, respectively. ${ }^{(20)}$ Although the protocols are variable, many centres currently practice goal-directed transfusion guided by laboratory results.

Surgical exploration should be reserved for cases with an uncertain diagnosis or late complications, such as active intraabdominal haemorrhage, suspected bowel ischaemia or perforation, peritonitis, and intestinal obstruction that is not responding to conservative treatment strategies. ${ }^{(5)}$ Arterial embolisation may have a therapeutic role in spontaneous retroperitoneal, soft tissue or extremity (anterior abdominal wall, iliopsoas, gluteal and thigh regions) haemorrhage. ${ }^{(24)}$ Notwithstanding these, there is currently no robust guideline available to guide endovascular or surgical intervention. Our patient was successfully treated conservatively and recovered well with no significant sequelae or symptom recurrence at the last follow-up.

New-generation direct oral anticoagulants (DOACs) such as direct thrombin inhibitors (dabigatran) and direct factor Xa (FXa) inhibitors (rivaroxaban, apixaban and edoxaban) are reported to offer important safety advantages over vitamin K antagonists such as warfarin. Besides being as effective in preventing thromboembolism, novel DOACs are also associated with lower rates of lifethreatening and intracranial bleeding. ${ }^{(25)}$ In addition, DOACs may be administered in fixed doses without routine coagulation monitoring, except in cases of emergent bleeding, where laboratory measurement of blood levels or anticoagulant activity may be useful. Commonly available general coagulation tests (e.g. prothrombin time and activated partial thromboplastin time) have limitations in measuring the anticoagulant effects of DOACs. Specific assays such as dilute thrombin time, ecarin clotting time, ecarin chromogenic assays and chromogenic anti-FXa assays are commercially available but not widely implemented in clinical practice.

Strategies for managing bleeding with non-specific haemostatic agents differ between patients anticoagulated with DOACs and those with warfarin. In bleeding patients who have been anticoagulated with warfarin, using haemostatic products such as fresh frozen plasma (FFP) and prothrombin complex concentrate (PCCs) is useful in replenishing clotting factors. When vitamin $\mathrm{K}$ is added, the effects of FFP and PCCs are sustained. In comparison, vitamin $\mathrm{K}$ does not reverse the anticoagulant effect of DOACs. Instead, non-activated PCCs such as four-factor and three-factor PCCs may be effective in managing bleeding in patients who are anticoagulated with apixaban, dabigatran, rivaroxaban or edoxaban by increasing the levels of coagulation factors inhibited by DOACs. Administration of the procoagulant recombinant activated factor VIla has also been suggested for anticoagulated bleeding patients on dabigatran etexilate and apixaban. ${ }^{(25)}$ Currently, specific reversal agents are being developed for DOAC-induced bleeding complications. Some examples include: idarucizumab, a specific reversal agent for dabigatran; andexanet alfa, which is under evaluation for reversal of indirect or direct FXa inhibitors; and ciraparantag, which is under trial for reversal of DOACs and other anticoagulants.

In summary, intramural intestinal haematomas should be vigilantly searched for on imaging in any patient presenting with abdominal symptoms who is receiving anticoagulant therapy and particularly if the INR is excessively prolonged. In uncomplicated cases, prompt recognition facilitates early conservative treatment, which usually results in excellent patient outcomes and also avoids unnecessary surgical intervention. Similarly, awareness of a wide spectrum of bleeding manifestations induced by anticoagulation in other sites of the body, as described, and early recognition of their corresponding imaging features will enable both the referring clinician and reporting radiologist to execute timely interventions, which may result in lower patient morbidity and mortality.

ABSTRACT A 74-year-old woman receiving long-term anticoagulation with warfarin for chronic atrial fibrillation presented with severe acute abdominal pain, diarrhoea and vomiting. Initial laboratory workup revealed a deranged coagulation profile. Computed tomography of the abdomen and pelvis demonstrated spontaneous distal jejunal intramural haematoma with associated reactive ileus. No overt pneumatosis intestinalis, intraperitoneal free gas or haemoperitoneum was seen. Based on clinical and imaging findings, a diagnosis of over-anticoagulation complicated by small bowel intramural haematoma was made. The patient was managed non-operatively with analgesia, cessation of warfarin and reversal therapy with vitamin $\mathrm{K}$. Warfarin therapy was recommenced upon resolution of symptoms and optimisation of coagulation status. The clinical presentation, radiological features and overall management of anticoagulation-induced bleeding are further discussed in this article.

Keywords: anticoagulants, atrial fibrillation, haematoma, haemorrhage, warfarin 


\section{REFERENCES}

1. Sorbello MP, Utiyama EM, Parreira JG, Birolini D, Rasslan S. Spontaneous intramural small bowel haematoma induced by anticoagulant therapy: review and case report. Clinics (Sao Paulo) 2007; 62:785-90.

2. Jimenez J. Abdominal pain in a patient using warfarin. Postgrad Med J 1999 75:747-8.

3. Shaw PH, Ranganathan S, Gaines B. A spontaneous intramural hematoma of the bowel presenting as obstruction in a child receiving low-molecular-weight heparin. J Pediatr Hematol Oncol 2005; 27:558-60.

4. Abbas MA, Collins JM, Olden KW. Spontaneous intramural small-bowel hematoma: imaging findings and outcome. AJR Am J Roentgenol 2002; 179:1389-94.

5. Abdel Samie A, Theilmann L. Detection and management of spontaneous intramural small bowel hematoma secondary to anticoagulant therapy. Expert Rev Gastroenterol Hepatol 2012; 6:553-9.

6. Jaakkola S, Nuotio I, Kiviniemi TO, et al. Incidence and predictors of excessive warfarin anticoagulation in patients with atrial fibrillation-the EWA study. PLoS One 2017; 12:e0175975.

7. Abdel Samie A, Sun R, Huber A, Höpfner W, Theilmann L. Spontaneous intramural small-bowel hematoma secondary to anticoagulant therapy: a case series. Med Klin Intensivmed Notfmed 2013; 108:144-8.

8. Judd DR, Taybi H, King H. Intramural hematoma of the small bowel: a repor of two cases and a review of the literature. Arch Surg 1964; 89:527-35.

9. Chu YY, Sung KF, Ng SC, Cheng HT, Chiu CT. Achalasia combined with esophageal intramural hematoma: case report and literature review. World Gastroenterol 2010; 16:5391-4.

10. Augustin G, Smud D, Kinda E, et al. Intra-abdominal bleeding from a seromuscular tear of an ascending rectosigmoid intramural hematoma after stapled hemorrhoidopexy. Can J Surg 2009; 52:E14-5.

11. Dohan A, Darnige L, Sapoval M, Pellerin O. Spontaneous soft tissue hematomas. Diagn Interv Imaging 2015; 96:789-96.

12. Bevan P, Menon A, Bunton R. Spontaneous chest wall hematoma with dual antiplatelet therapy. Can J Cardiol 2014; 30:247.e1-2.

13. Moloney F, Murphy KP, Twomey M, O'Connor OJ, Maher MM. Haematuria: an imaging guide. Adv Urol 2014; 2014:414125.

14. Ibrahim W, Mohamed A, Sheikh M, et al. Antiplatelet therapy and spontaneous retroperitoneal hematoma: a case report and literature review. Am J Case Rep 2017; 18:85-9.

15. Sunga KL, Bellolio MF, Gilmore RM, Cabrera D. Spontaneous retroperitoneal hematoma: etiology, characteristics, management, and outcome. J Emerg Med 2012; 43:e157-61.

16. Cervera A, Amaro S, Chamorro A. Oral anticoagulant-associated intracerebral hemorrhage. J Neurol 2012; 259:212-24.

17. Hemphill JC 3rd, Greenburg SM, Anderson CS, et al; American Heart Association Stroke Council; Council on Cardiovascular and Stroke Nursing; Council on Clinical Cardiology. Guidelines for the management of spontaneous intracerebral hemorrhage: a guideline for healthcare professionals from the American Heart Association/American Stroke Association. Stroke 2015; 46:2032-60.

18. Cheng J, Vemula N, Gendler S. Small bowel obstruction caused by intramural hemorrhage secondary to anticoagulant therapy. Acta Gastroenterol Belg 2008; 71:342-4.

19. Kwon $\mathrm{Cl}, \mathrm{Ko} \mathrm{KH}$, Kim HY, et al. Bowel obstruction caused by an intramural duodenal hematoma: a case report of endoscopic incision and drainage. J Korean Med Sci 2009; 24:179-83.

20. Tomaselli GF, Mahaffey KW, Cuker A, et al. 2017 ACC expert consensus decision pathway on management of bleeding in patients on oral anticoagulants: a report of the American College of Cardiology Task Force on expert consensus decision pathways. J Am Coll Cardiol 2017; 70:3042-67.

21. Chai-Adisaksopha $C$, Hillis $C$, Isayama T, et al. Mortality outcomes in patients receiving direct oral anticoagulants: a systematic review and meta-analysis of randomized controlled trials. J Thromb Haemost 2015; 13:2012-20.

22. Qureshi W, Mittal C, Patsias I, et al. Restarting anticoagulation and outcomes after major gastrointestinal bleeding in atrial fibrillation. Am J Cardiol 2014; 113:662-8

23. Carson JL, Guyatt G, Heddle NM, et al. Clinical practice guidelines from the AABB: red blood cell transfusion thresholds and storage. JAMA 2016; 316:2025-35

24. Dohan A, Sapoval M, Chousterman BG, et al. Spontaneous soft-tissue hemorrhage in anticoagulated patients: safety and efficacy of embolization. AJR Am J Roentgenol 2015; 204:1303-10.

25. Eikelboom J, Merli G. Bleeding with direct oral anticoagulants vs warfarin: clinical experience. Am J Med 2016; 129:S33-40 


\section{SINGAPORE MEDICAL COUNCIL CATEGORY 3B CME PROGRAMME} (Code SMJ 201911B)

Question 1. Regarding anticoagulant-induced bleeding:

(a) The transverse colon and jejunum are common sites for bleeding in the gastrointestinal tract.

(b) Blunt abdominal trauma is an aetiological factor for duodenal haematoma.

(c) Novel direct oral anticoagulants (DOACs) such as rivaroxaban have a higher risk of intracranial bleeding than warfarin.

(d) Underlying amyloid angiopathy is a risk factor for intracranial re-bleeding after restarting anticoagulation.

Question 2. Regarding imaging of gastrointestinal intramural haematoma:

(a) Radiological features on computed tomography $(\mathrm{CT})$ include circumferential bowel wall thickening and intramural hyperdensity.

(b) Complete resolution of imaging findings usually occurs within two months after the onset of symptoms.

(c) The concentric ring sign on magnetic resonance imaging is due to the paramagnetic properties of iron in the haemoglobin degradation products within the haematoma.

(d) A non-contrast CT scan may be performed before the acquisition of contrast-enhanced images to demonstrate the presence of any intramural haemorrhage, which may be masked by contrast enhancement.

Question 3. The risk factors for developing small bowel haematomas include:
(a) Haemophilia.
(b) Over-anticoagulation.
(c) Leukaemia.
(d) Vasculitis.

Question 4. Which of the following applies to the management strategies of anticoagulant-induced bleeding?

(a) Discontinuation of the anticoagulant and use of the relevant reversal agent.

(b) Transfusion of blood products when there is haemodynamic compromise or a haemoglobin drop of $\geq 2 \mathrm{~g} / \mathrm{dL}$.

(c) Therapeutic arterial embolisation does not have a role in spontaneous retroperitoneal, soft tissue or extremity haemorrhage.

(d) Surgery is indicated for all cases of anticoagulant-induced gastrointestinal haemorrhage.

Question 5. Regarding new-generation DOACs:

(a) DOACs are as effective as vitamin $\mathrm{K}$ antagonists in preventing thromboembolism.

(b) Chromogenic anti-factor Xa is routinely used as an assay to measure the anticoagulant effect of warfarin.

(c) Non-activated prothrombin complex concentrates may be used in the management of bleeding in patients on DOAC.

(d) Vitamin $\mathrm{K}$ does not reverse the anticoagulant effect of DOACs.

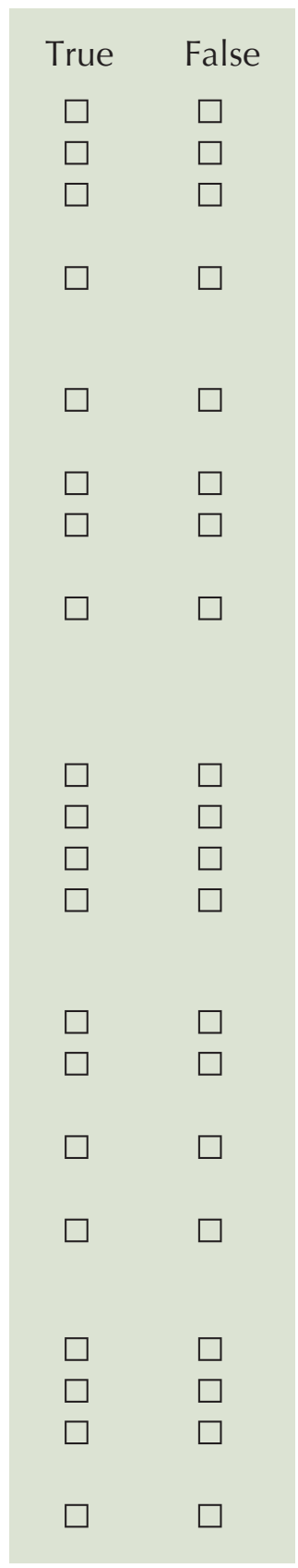

\section{Doctor's particulars:}

Name in full:

MCR no.:

Specialty:

Email:

\section{SUBMISSION INSTRUCTIONS:}

Visit the SMJ website: http://www.smj.org.sg/current-issue and select the appropriate quiz. You will be redirected to the SMA login page.

For SMA member: (1) Log in with your username and password (if you do not know your password, please click on 'Forgot your password?'). (2) Select your answers for each quiz and click 'Submit'.

For non-SMA member: (1) Create an SMJ CME account, or log in with your SMJ CME username and password (for returning users). (2) Make payment of SGD 21.40 (inclusive of $7 \%$ GST) via PayPal to access this month's quizzes. (3) Select your answers for each quiz and click 'Submit'.

RESULTS:

(1) Answers will be published online in the SMI January 2020 issue. (2) The MCR numbers of successful candidates will be posted online at the SMI website by 7 January 2020. (3) Passing mark is $60 \%$. No mark will be deducted for incorrect answers. (4) The SMJ editorial office will submit the list of successful candidates to the Singapore Medical Council. (5) One CME point is awarded for successful candidates. (6) SMC credits CME points according to the month of publication of the CME article (i.e. points awarded for a quiz published in the December 2017 issue will be credited for the month of December 2017, even if the deadline is in January 2018).

Deadline for submission (November 2019 SMJ 3B CME programme): 12 noon, 31 December 2019. 